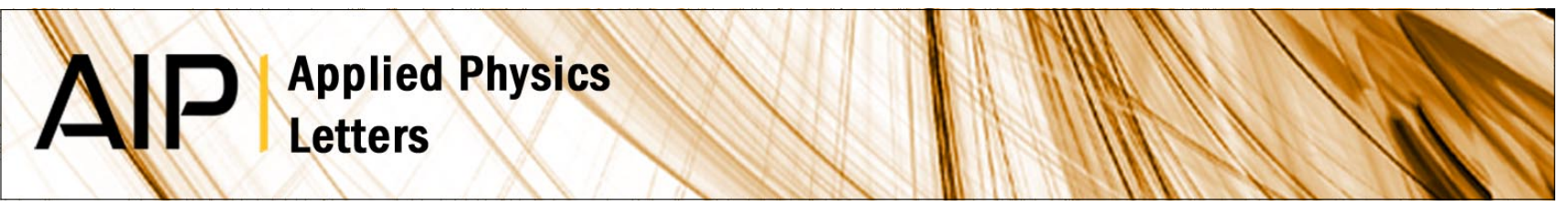

\title{
Quantitative atom-resolved force gradient imaging using noncontact atomic force microscopy
}

Ahmet Oral, Ralph A. Grimble, H. Özgür Özer, Peter M. Hoffmann, and John B. Pethica

Citation: Appl. Phys. Lett. 79, 1915 (2001); doi: 10.1063/1.1389785

View online: http://dx.doi.org/10.1063/1.1389785

View Table of Contents: http://apl.aip.org/resource/1/APPLAB/v79/i12

Published by the American Institute of Physics.

Additional information on Appl. Phys. Lett.

Journal Homepage: http://apl.aip.org/

Journal Information: http://apl.aip.org/about/about_the_journal

Top downloads: http://apl.aip.org/features/most_downloaded

Information for Authors: http://apl.aip.org/authors

\section{ADVERTISEMENT}

\section{AIP | Applied Physics Letters}

MIPI I IO III

Accepting Submissions in

Biophysics and Bio-Inspired Systems 


\title{
Quantitative atom-resolved force gradient imaging using noncontact atomic force microscopy
}

\author{
Ahmet Oral, ${ }^{a), b)}$ Ralph A. Grimble, H. Özgür Özer, ${ }^{a)}$ Peter M. Hoffmann, and \\ John B. Pethica \\ Department of Materials, University of Oxford, Parks Road, Oxford OX1 3PH, United Kingdom
}

(Received 4 April 2001; accepted for publication 11 June 2001)

\begin{abstract}
Quantitative force gradient images are obtained using a sub-angström amplitude, off-resonance lever oscillation method during scanning tunneling microscopy imaging. We report the direct observation of short-range bonds, and the measured short-range force interaction agrees well in magnitude and length scale with theoretical predictions for single bonds. Atomic resolution is shown to be associated with the presence of a prominent short-range contribution to the total force interaction. It is shown that the background longer-range interaction, whose relative magnitude depends on the tip structure, has a significant effect on the contrast observed at the atomic scale. (C) 2001 American Institute of Physics. [DOI: 10.1063/1.1389785]
\end{abstract}

The atomic force microscope $(\mathrm{AFM})^{1}$ was originally devised as an analog of the scanning tunneling microscope (STM), using forces rather than the tunnel current between tip and surface atoms to generate atomic resolution images. Forces associated with short-range bonding have, like the tunnel current, an exponential dependence on distance, ${ }^{2,3}$ making atomic resolution possible. However, most of the actual applications of AFM have been at lower than atomic resolution because of the extreme sensitivity required to resolve single bonds, and the instability of soft levers against the high force gradients of short-range interactions. Atomic resolution AFM has been achieved only recently using resonant cantilevers with large, $\sim 100 \AA$ oscillation amplitudes in ultrahigh vacuum (UHV). ${ }^{4-6}$ This technique has also been used to measure the force interactions between the tip and sample, ${ }^{7}$ but the oscillation amplitudes employed are much greater than the interaction range. Hence the interaction has to be inferred by mathematical deconvolution, which assumes both single valuedness of the interaction and the absence of dissipation processes during the measurement. There have been more direct measurements of the interaction. Dürig et al. ${ }^{8}$ observed short-range interactions using an Ir sample, but their work did not involve imaging. Other direct measurements have shown unexpectedly large length scales for the interaction potentials ${ }^{9,10}$ implying the dominance of forces other than atomic local bonding. In this letter, we report the direct observation of short-range bonds, and we show that they are indeed associated with atomic resolution in AFM as predicted by theory. ${ }^{11}$

Experiments are performed with a specially constructed high force resolution AFM/STM operating in UHV. ${ }^{12}$ The force gradient between the tip and sample is measured by vibrating the cantilever base at a frequency well below the resonance, with a very small oscillation amplitude of typically $0.25-0.5 \AA$ A peak to peak. Excellent displacement sensitivity is thus very important. An all-fiber interferometer is

${ }^{a)}$ Present address: Department of Physics, Bilkent University, 06533 Ankara, Turkey.

b)Electronic mail: ahmet@fen.bilkent.edu.tr used to detect the cantilever deflections, ${ }^{13}$ and the reflectivities of the fiber end and the lever are tailored to enhance the cavity's finesse. The system has a $3 \times 10^{-4} \AA / \sqrt{ } \mathrm{Hz}$ noise floor, which enables $<1 \mathrm{~N} / \mathrm{m}$ stiffness changes to be observed with sub- $\AA$ oscillation amplitudes using reasonable integration times. The amplitude at the tip, which is altered by the tip-surface interaction, is detected from the interferometer output using a lock-in amplifier. The interaction stiffness (negative of the force gradient) between the tip and sample $k_{\text {int }}$ can be obtained from the simple relation ${ }^{14}$

$$
k_{\mathrm{int}}=-\frac{d F}{d z}=k_{0}\left(\frac{A_{0}}{A}-1\right),
$$

where $k_{0}, A_{0}$ and $A$ are the lever stiffness, free oscillation amplitude and measured oscillation amplitude of the lever, respectively. The use of very small amplitudes has two advantages. First, the interaction is determined at a single separation, and is thus easily correlated with the simultaneously observed tunnel current. Second, the interaction is effectively linearized at that point, and its value is directly found from a simple spring analysis, as in Eq. (1). This contrasts with large amplitude techniques, where the nonlinear nature of the interaction necessitates deconvolution of frequency shift data and the use of certain a priori assumptions about the nature of the interaction. Most literature reports confine themselves to reporting the frequency shifts of the resonant lever rather than the actual interaction force gradients.

The cantilevers are made from flattened and polished tungsten wires whose ends are bent and then etched to obtain sharp tips. The levers have stiffnesses in the range of 100$250 \mathrm{~N} / \mathrm{m}$ in order to be safely above the typical maximum (negative) interaction stiffness and thus avoid the snap-in instability at smaller separations. The stiffness of the levers is determined by measuring the thermal oscillation amplitude and also independently by a laser interferometer and a calibrated test counterlever and a nanoindenter. The levers have $11-20 \mathrm{kHz}$ resonant frequencies and quality factors, $Q$, of 600-3000. The piezo motions are calibrated using the fiber interferometer. The experiments are performed in an UHV system with a base pressure of $\sim 5 \times 10^{-11}$ mbar. The 


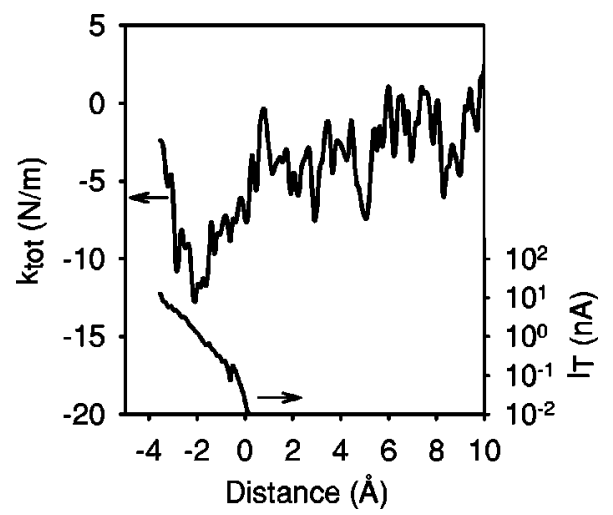

FIG. 1. Force gradient $\left(A_{0}=0.25 \AA_{\mathrm{pp}}\right.$ and $\left.k_{0}=180 \mathrm{~N} / \mathrm{m}\right)$ and tunnel current as a function of tip-sample separation on a $\mathrm{Si}(111)(7 \times 7)$ surface. Typical result for tips having a significant contribution of short-range terms to the total measured force gradient. The length scale of the short-range interaction is found to be $1.1 \AA$ from a curve fit after subtracting the long-range terms and accounting for the lever and tip relaxation (Ref. 15).

$\operatorname{Si}(111)(7 \times 7)$ surface is prepared in situ in the standard manner, finishing by flashing the sample to $1080^{\circ} \mathrm{C}$. The microscope is stable for long periods and we can record images overnight automatically.

The tip is brought close to the surface while monitoring the tunnel current and the force gradient simultaneously. For some tips we see primarily long-range interactions, and the forces are already significant before any tunnel current is observed. This is likely due to van der Waals or perhaps electrostatic forces which, because of either the tip shape or chemical nature of the tip material, mask the short-range, highly local forces. ${ }^{15}$ However, with other tips we observe behavior like that shown in Fig. 1. The tunnel current in these cases rises at, or before, the onset of an observable force interaction, an indication that the longer-range forces are not as dominant. The tunnel barrier $\Phi=d \ln I / d z$ is $\sim 2 \mathrm{eV}$ at larger separations and then falls at smaller separations, as has been observed previously. ${ }^{16}$ Note that this occurs when the force interaction becomes strong, and so perturbation of the local atom positions might be occurring as well as electronic barrier quenching. ${ }^{17}$ The whole approach and retraction process remains reversible provided the separation is not reduced beyond the point at which the measured stiffness becomes positive (i.e., the point of inflection on the binding energy curve). The curve shown here is a single approach curve and is not averaged over several different measurements. The observable residual noise is due to the use of very low $\left(0.25 \AA_{\mathrm{pp}}\right)$ oscillation amplitude as well as to the relatively short $(30 \mathrm{~ms})$ time constant used in the lock-in amplifier. After subtracting the (in this case relatively weak) long-range components and taking the relaxation of the lever and tip into account, ${ }^{15}$ we have fitted the force gradient curves to a universal bonding potential ${ }^{18}$ and obtain a decay length scale for tip interaction of $1.1 \AA$ and a minimum bonding energy of $3.7 \mathrm{eV}$ which is in good agreement with the $a b$ initio calculations. ${ }^{19}$ The maximum tensile stiffness in Fig. 1 is $\sim 13 \mathrm{~N} / \mathrm{m}$ and integration of the curve gives $\sim 3 \mathrm{nN}$ maximum force. Of this about $5 \mathrm{~N} / \mathrm{m}$ and $1.2 \mathrm{nN}$ (i.e., about $40 \%$ ) are due to short-range interactions. We are thus measuring the short-range potentials responsible for a single
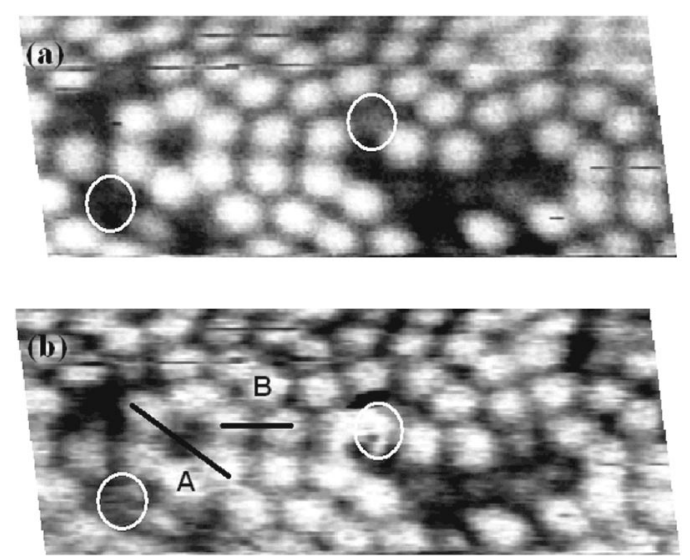

FIG. 2. Simultaneous STM image (a) and force gradient image (b) of a $\operatorname{Si}(111)(7 \times 7)$ surface. Measurement conditions: $V_{\text {tip }}=-1 \mathrm{~V}, I_{T}=1 \mathrm{nA}$, $A_{0}=0.5 \AA_{\mathrm{pp}}$ and $k_{0}=116 \mathrm{~N} / \mathrm{m}$. In image (b), darker areas indicate more negative (attractive) values of the interaction stiffness.

strong interatomic bond. We shall see next that this is confirmed in the force gradient imaging.

Figure 2 shows simultaneously acquired STM and force gradient images. An important observation is that we were only able to obtain atomic resolution in the force gradient images (Fig. 2) when the tip-surface interaction exhibited significant short-range contributions to the total force gradient, i.e., when we have a tip which exhibits a short overall characteristic length scale, approaching that shown in Fig. 1. This confirms the theoretical expectation that short-range forces are required for atom-resolved AFM. ${ }^{11}$ Note that the STM topography and force gradient images, although similar, do not show exactly the same features. For example, the areas circled in Fig. 2 show that apparently missing adatoms in the STM image give a prominent contrast in the AFM image. This could be due to the presence of an adsorbate atom with a low STM density of states at this site, which would nevertheless contribute to the force. The corrugation from adatom to adatom via a corner hole (line $\mathbf{A}$ ) and from adatom to adatom via a restatom (line $\mathbf{B}$ ) are 12 and $4.5 \mathrm{~N} / \mathrm{m}$, respectively. The darker shading indicates higher attractive stiffness in the force gradient images and therefore the corner holes and restatoms actually have higher attractive stiffness than the adatoms, with the corner holes having the most attractive stiffness of all three sites. The force gradient contrast is always found to increase with decreasing separation (higher tunnel currents). We have also observed similar general behavior on $\mathrm{Si}(100)(2 \times 1)$. A change of tip during imaging reveals an even more complex relationship, as Fig. 3 shows. The tip changed spontaneously halfway up the image, resulting in a reduced STM contrast in the upper half. At the same time, the force gradient corrugation from adatom to adatom via a corner hole (line A) decreased from $11 \mathrm{~N} / \mathrm{m}$ before the tip change to $8.4 \mathrm{~N} / \mathrm{m}$ afterwards. However, the corrugation from adatom to adatom via a restatom (line $\mathbf{B}$ ) actually increased from 5.2 to $6.9 \mathrm{~N} / \mathrm{m}$. The average value of absolute stiffnesses measured at all the various sites became more negative (i.e., more attractive) by about $3-5 \mathrm{~N} / \mathrm{m}$.

Calculations by Pérez et al. ${ }^{19}$ indicate that the force gradient magnitude over the holes should be less than that over the adatoms, the opposite of what we observe. However, this assumes a laterally flat tip trajectory, whereas in reality the 


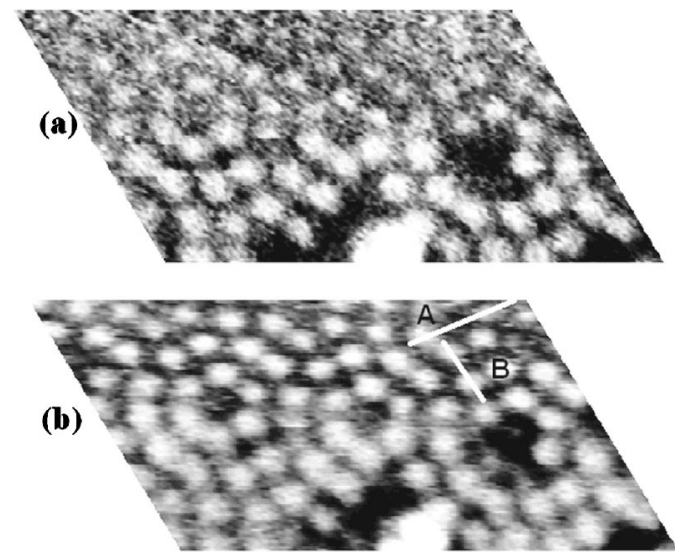

FIG. 3. Simultaneous STM image (a) and force gradient image (b) of a $\mathrm{Si}(111)(7 \times 7)$ surface sample where the tip changes halfway up the image. $V_{\text {tip }}=-1 \mathrm{~V}, I_{T}=2 \mathrm{nA}, A_{0}=0.5 \AA_{\mathrm{pp}}$ and $k_{0}=116 \mathrm{~N} / \mathrm{m}$. Darker areas in (b) indiate more negative values in the interaction stiffness.

tip atom follows a corrugated path during imaging. It might also be displaced relative to the more distant (i.e., experimentally observed) parts of the tip in response to force corrugations. We can explain why the corner holes and restatoms show higher attractive stiffness as follows. As already noted, there will in general be some background (van der Waals) force between the tip and sample. As the tip follows the STM contours, this background term will give an additional attractive force gradient where the tip moves closer to the surface, and will thus be largest over the corner holes. To calculate this effect we first take an empirical van der Waals interaction term

$$
\left(k_{\mathrm{vdW}}=-\frac{A_{H}}{2} \frac{R^{2}}{\left(d-d_{0}\right)^{4}}\right)
$$

(Ref. 15) (where $d_{0}$ is an offset distance allowing the local value of the gap $d$ to be related to the defining center position of the background force, $R$ is the van der Waals radius of the tip, and $A_{H}$ is the Hamaker constant, taken to be 3 $\times 10^{-19} \mathrm{~J}$ ). The parameters are fitted from the long-range parts of the observed interactions, as described in detail elsewhere. ${ }^{15}$ Now, the local (short-range) potentials of the atom at each site (adatom, restatom and corner hole) are taken from the simulations of Pérez et al. ${ }^{19}$ They are then shifted from their correct lattice positions by appropriate distances to allow for the actual measured displacement (STM corrugation) of the tip over each site (1.1 $\AA$ for corner holes and $0.3 \AA$ for restatoms in Fig. 2). This gives the correct short-range force gradient at each site for the actual tip path. Then we add the force gradient of the long-range potential, again allowing for the actual displacement path of the tip. For realistic (and observed) long-range terms, this gives both the sign and magnitude of the force gradient corrugations that we actually observe.

Consideration of the defects in Fig. 2 shows that the force corrugation is not simply related to the STM corrugation, and that changes in short-range forces have a major effect on the images. This is particularly evident in trying to model the effect of the tip change in Fig. 3. Without changing the short-range potentials, it is only possible to cause the corner hole corrugation to decrease and the adatom corrugation to remain constant (let alone increase) if both the separation and the background potential term is decreased. Neither of these is very likely, since the STM corrugation actually decreases (implying larger local separation) and the required large scale tip shape change would be expected to cause more serious image disruption. We are obliged to conclude that the chemical nature of the tip atom has changed and altered its bonding to the individual surface sites, and hence the image corrugation.

We have shown that, using ultrasmall oscillation amplitudes, atom-resolved quantitative AFM imaging and direct force spectroscopy are possible. Short-range interactions are crucial for imaging, but long-range terms need to be incorporated to explain the image corrugations observed. The results clearly show that even in normal STM imaging, there are quite strong forces acting. This could lead to significant movement of atoms (including on the tip) ${ }^{20}$ and hence seriously affect STM image contrast in general experiments. Our results also show that the relation between current and force, of importance for potential nanoscale devices, is complex on the single atom scale. However, the combination of AFM and STM could clarify the subject in the future.

This work was supported by the Royal Society Paul Fund and by EPSRC. One of the authors (A.O.) thanks the British Council for travel support and a second author (H.Ö.Ö.) thanks TUBITAK for a BDP scholarship.

${ }^{1}$ G. Binnig, C. F. Quate, and Ch. Gerber, Phys. Rev. Lett. 56, 930 (1986).

${ }^{2}$ C. J. Chen, Introduction to Scanning Tunneling Microscopy (Oxford University Press, Oxford, 1993), pp. 185-193.

${ }^{3}$ S. Ciraci, in Scanning Tunneling Microscopy, edited by R. Wiesendanger and H.-J. Güntherodt (Springer, Berlin, 1996), Vol. 3, Chap. 8.

${ }^{4}$ F. J. Giessibl, Science 267, 69 (1995).

${ }^{5}$ H. Ueyama, M. Ohta, Y. Sugawara, and S. Morita, Jpn. J. Appl. Phys., Part 2 34, L1086 (1995).

${ }^{6}$ S. Kitamura and M. Iwatsuki, Jpn. J. Appl. Phys., Part 2 34, L145 (1995).

${ }^{7}$ M. A. Lantz, H. J. Hug, P. J. A. van Schendel, R. Hoffmann, S. Martin, A. Baratoff, A. Abdurixit, H.-J. Güntherodt, and Ch. Gerber, Phys. Rev. Lett. 84, $2642(2000)$

${ }^{8}$ U. Dürig, O. Züger, and D. W. Pohl, Phys. Rev. Lett. 65, 349 (1990).

${ }^{9}$ G. Cross, A. Schirmeisen, A. Stalder, P. Grütter, M. Tschudy, and U. Dürig, Phys. Rev. Lett. 80, 4685 (1998).

${ }^{10}$ S. P. Jarvis, H. Yamada, S.-I. Yamamoto, H. Tokumoto, and J. B. Pethica, Nature (London) 384, 247 (1996).

${ }^{11}$ R. Pérez, M. C. Payne, I. Stich, and K. Terakura, Appl. Surf. Sci. 123, 249 (1998).

${ }^{12}$ A. Oral, R. A. Grimble, H. Ö. Özer, and J. B. Pethica (unpublished).

${ }^{13}$ D. Rugar, H. J. Mamin, and P. Guethner, Appl. Phys. Lett. 55, 2588 (1989)

${ }^{14}$ S. P. Jarvis, A. Oral, T. P. Weihs, and J. B. Pethica, Rev. Sci. Instrum. 64, 3515 (1993)

${ }^{15}$ P. M. Hoffmann, A. Oral, R. A. Grimble, H. Ö. Özer, S. Jeffery, and J. B. Pethica, Proc. R. Soc. London, Ser. A 457, 1161 (2001).

${ }^{16}$ U. Dürig, J. K. Gimzewski, and D. W. Pohl, Phys. Rev. Lett. 57, 2403 (1986).

${ }^{17}$ L. Olesen, M. Brandbyge, M. R. Sørensen, K. W. Jacobsen, E. Lægsgaard, I. Stensgaard, and F. Besenbacher, Phys. Rev. Lett. 76, 1485 (1996).

${ }^{18}$ A. P. Sutton, Electronic Structure of Materials (Oxford University Press, Oxford, 1993), pp. 1-6.

${ }^{19}$ R. Pérez, I. Stich, M. C. Payne, and K. Terakura, Phys. Rev. B 58, 10835 (1998).

${ }^{20}$ A. R. H. Clarke, J. B. Pethica, J. A. Nieminen, F. Besenbacher, E. Lægsgaard, and I. Stensgaard, Phys. Rev. Lett. 76, 1276 (1996). 